\title{
Wide Area Integrated Maritime Surveillance: An Updated Architecture with Data Fusion
}

\author{
Z. Ding, G. Kannappan \\ Raytheon Canada Ltd. \\ K. Benameur \\ Defence Research and Development Canada \\ T. Kirubarajan \\ McMaster University, Canada \\ M. Farooq \\ Royal Military College, Canada
}

\begin{abstract}
An architecture has been proposed for an integrated maritime surveillance (IMS) system to support Canada's defense efforts in a network-centric or information-centric surveillance of the Canadian coasts, where many sensors, for example, HF radar, surface search radar, Automatic Dependent Surveillance (ADS) and imaging radar are used to gather information about the targets of interest. Typically, a realistic IMS system is a distributed one where the information processing is carried out in a number of locations and at different levels (e.g., local IMS, regional IMS or national surveillance center). This paper will discuss $I M S$, propose an architecture for an IMS system and give some specifications for the proposed IMS system. The paper will describe the implemented data fusion engine. Desirable features are also given in the paper.
\end{abstract}

Keywords: IMS, data fusion, high frequency surface wave radar, target tracking, architecture

\section{Integrated Maritime Surveillance}

Protection of a nation's sovereignty and effective management of the development and exploitation of natural resources within the 200 nautical mile (nm) Exclusive Economic Zone (EEZ) place new demands on the organizations traditionally tasked with maritime surveillance and enforcement $[1,2,3]$. As a general principle, the level of surveillance that is required at any given time depends on the perceived threat. The ideal surveillance system must be capable of normal, day-to-day operation for the lowest possible cost yet have the resources to respond decisively and economically when required.

The provision of enhanced levels of surveillance will play a key role in the recovery of Canada's East coast fishing industry devastated by over fishing and a 1992 government imposed moratorium banning fishing. Likewise, most other maritime nations face comparable requirements in ensuring that their future economic development and the protection of offshore natural resources are not prejudiced.

The successful management of inshore and offshore fisheries, exploitation of oil and natural gas resources, undersea mining, tourism, and similar ventures, not forgetting environmental protection and disaster management, will ultimately depend on achieving significant improvements in the effectiveness of surveillance infrastructures world wide. Furthermore, such improvements must be achievable without significant increases in 
spending profiles as, is the case in many countries, additional funding will be increasingly more difficult to secure.

Surveillance of activity in and beyond the EEZ is but one element in any nation's approach to the enforcement of national security and the management of trade, resource development and transportation. With the increasing availability and deployment of sophisticated information management systems, the trend is towards the implementation of systems to associate information at ever higher levels and produce a common national "big picture" against which all activities will be coordinated and managed.

The advent of shore based High Frequency Surface Wave Radars (HFSWR) permits continuous, cost effective, surveillance to beyond the $200 \mathrm{~nm}$ limit [1-6]. Surface wave radar provides the background picture by continuously tracking both surface and low level air targets in the area under surveillance. However, the tracking of targets is only of real value if those targets can be identified. Fusing the HFSWR radar picture with inputs from other existing surveillance systems supports target classification and identification. Existing systems can include mandatory reports from vessels entering and leaving the EEZ, reports from patrol vessels and aircraft, the interpretation of imaging satellite data, coastal radar data, etc. This is the concept called Integrated Maritime Surveillance (IMS).

This paper describes an IMS system based on HFSWR for monitoring surface and low-level air activity within the $200 \mathrm{~nm}$ EEZ. The system is designed to help authorities locate and identify such illegal activities as drug trafficking, smuggling, piracy, illegal fishing and illegal immigration. In addition, the system may be used for tracking icebergs, environmental protection, search and rescue, resource protection, sovereignty monitoring and remote sensing of ocean surface currents and winds. Based on an existing HFSWR system, an architecture is proposed to implement an IMS system. Both local IMS (LIMS) and regional IMS (RIMS) will be given. Main specifications for design will be given.

\section{An Architecture for an Integrated Maritime Surveillance (IMS)}

The ability to continuously track and identify ships and airplanes throughout the EEZ was one of the principal goals in the development of Raytheon's shore based HFSWR. While HFSWR has the capability to track and broadly classify airplanes and ships (small, medium and large), it cannot establish positive identification. Consequently, the development also addressed the requirement to automatically associate radar data with data from a variety of other sensors and systems to resolve this shortcoming. This will lead to the successful implementation of a MultiSensor Fusion Processor and ultimately an IMS system, a system built with the following elements:

Long Range Surface Wave Radar: For example, the Raytheon SWR-503 HFSWR will be the primary sensor of the IMS system.

Automatic Dependent Surveillance (ADS): Vessels and aircraft operating with ADS systems transmit position and identification information on a regular schedule over pre-assigned terrestrial and satellite communications channels.

Other Sensors and Information Sources: RadarSat provides image information. Registration database provides information for all international ships. Microwave radar provides short range surveillance.

Data Fusion Engine: A central data fusion engine automatically correlates target detections or tracks derived from long range radar systems and other complementary real-time sensors.

Data Distribution Server: The Data Distribution Server will provide all required services for authorized clients.

IMS Administration: Provides day-to-day administration like configuration, running or stopping IMS, user accounts and security, managing storage, managing transaction logs, making and restoring backups etc. The display client of the IMS administration provides presentation for customers to view the surveillance picture and to take authorized actions. 
Communications Infrastructure of the IMS:

Provides communication framework.

Wide Area Surveillance: The system should cover a surveillance area as wide as is needed. For IMS, it means that we should have local IMS (LIMS) systems and regional IMS (RIMS) systems. A local IMS system is a system with a group of HFSWRs and other sensors which provide overlapped information. Two or more geographically separated LIMS systems form a regional IMS system when a surveillance center is required to coordinate some LIMS systems.

Considering the above elements and the existing components of the IMS system, an architecture for an IMS system can be constructed as follows:

\section{An Architecture for an IMS System}

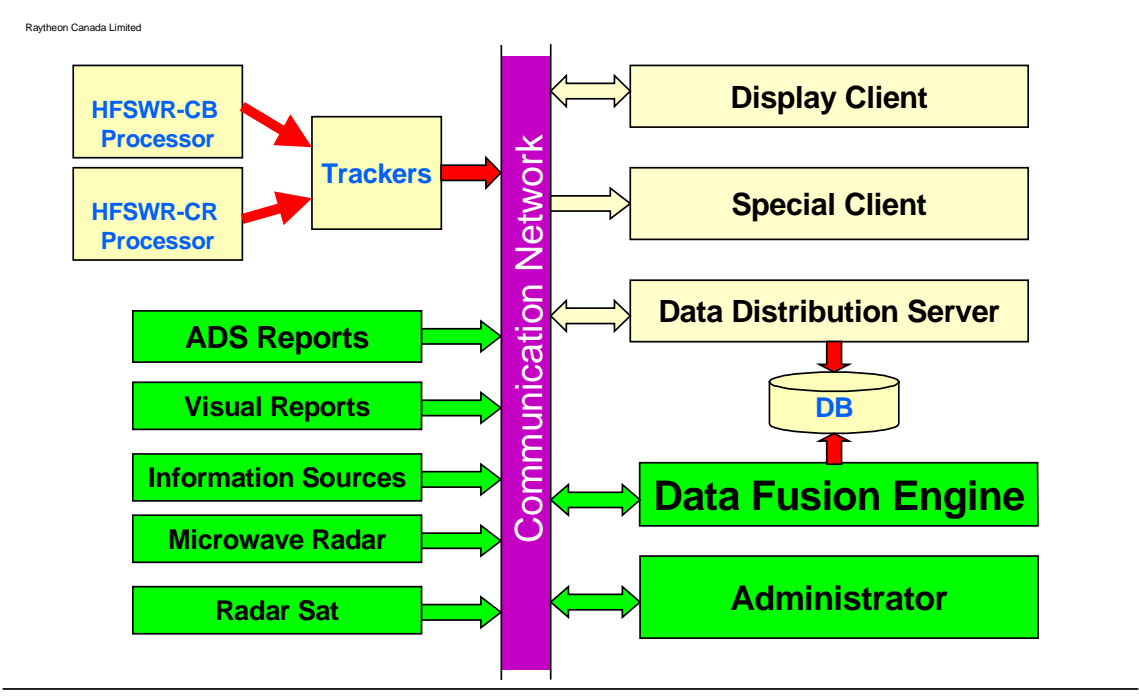

Figure 1: The Proposed IMS Architecture

where HFSWR-CB and HFSWR-CR refer to the two HFSWR at Cape Bonavista and Cape Race. The special clients means clients who need special services, for example, a classified client who needs data through secure data link.

\section{Data Fusion Engine}

The data fusion engine is the core of the IMS system. By means of fusion, different sources of information are combined to improve the existing surveillance capability. The most obvious illustration of fusion is the use of various sensors typically to detect a target or to build a layered picture.
Fusion is useful for improving several objectives such as detection, identification, tracking, change detection, decision making, etc. Using an

efficient fusion scheme, one may expect significant advantages as:

1. Improved confidence in decisions due to the use of complementary information (e.g. silhouette of objects from visible image, active/non-active status from infrared image, speed and range from radar, etc.)

2. Improved performance against countermeasures (it is very hard to camouflage an object in all possible wavebands)

3. Improved performance in adverse environmental conditions. Typically smoke or fog cause bad visible contrast and some 
weather conditions (rain) cause low thermal contrast, combining both types of sensors should give better overall performance.

Fusion processes are often categorized as low, intermediate or high level fusion depending on the processing stage at which fusion takes place.

Low level fusion combines several sources of raw data to produce output information that is expected to be more informative and synthetic than the inputs. This level of fusion aims at fusing 'relevant information' from the different sensors.

Intermediate level fusion combines various features. Those features may come from several raw data sources (several sensors, different moments, etc.) or from the same raw data.

High level fusion combines decisions coming from several experts. By extension, one speaks of decision fusion even if the experts return a confidence (score) and not a decision.

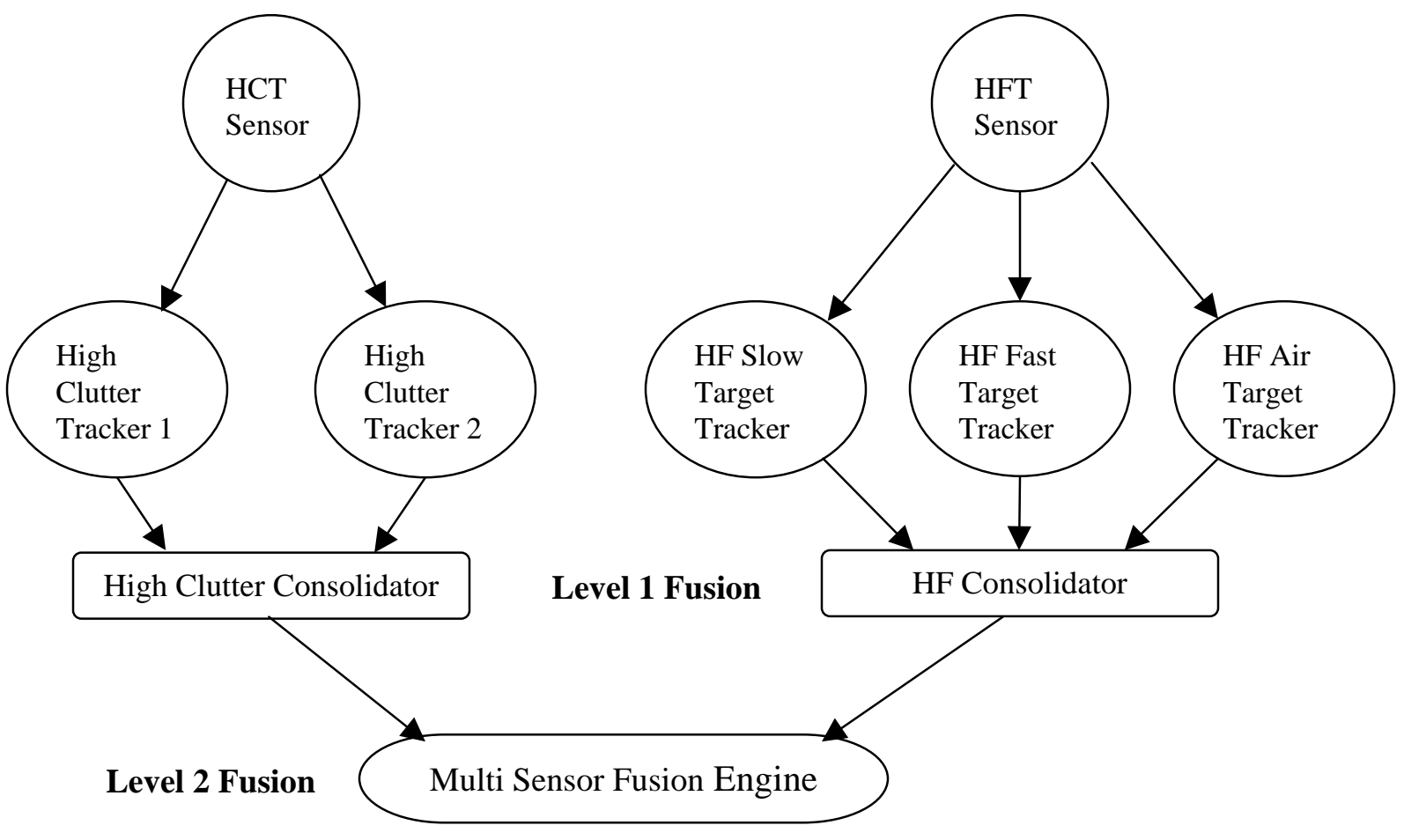

Figure 2: Two Level Data Fusion Engine

In practical problems, the applied fusion procedure is often a combination of the previously mentioned three levels. In the proposed IMS system, the data fusion will be at low and intermediate levels, with the capability to incorporate a high level component. Some of the related contents can be found in $[1,4,5,8$ 13].

The multi-sensor data fusion engine we proposed and implemented is a two level data fusion. Level one fusion will occur on the radar side. For the High Clutter Tracker (HCT), a small target detection and tracking system for mircowave radar, this involves the consolidation of all the trackers that are receiving data from the same sensor but are possibly processing it

differently due to different system parameters. Similarly, for HFSWR system, the slow, fast, and air trackers will have to be consolidated. Once each system performs level one fusion it will send the consolidated data to the multi sensor fusion engine where second level fusion will take over the multi-sensor track-track fusion.

Figure 2 shows a schematic diagram of the L1 and L2 fusion, involving one HCT and one HFSWR sensor. 
The advantage of this scheme is that it will keep the HCT system virtually the same. The only modification will be the addition of a module to send the data to the fusion engine. Also it will keep the HCT and HFSWR systems available for stand alone running.

\section{COMMUNICATION:}

The communication between the sensors and the fusion engine is accomplished by using TCP/IP as the transfer protocol and connection oriented socket. TCP/IP is used, as it is a more reliable protocol (as opposed to UDP). In order to minimize the number of open ports on the fusion system a single port is used for each type of sensor (as opposed to using a new port for every sensor). These ports can be defined through the fusion configuration file. The ones that are currently used for testing are as follows:

- $\quad$ HCT port: 60000

HFT port: 60001

ADS port: 60002

At start time, a $\mathrm{C}++$ procedure main() will initialize three threads, one for each type of sensor. All the threads will start with the "port listen' function, but each will take a different parameter (this parameter will specify the port to listen on). When one of these three threads detects an incoming connection, it spawns another thread that will receive the data and filter it into a structure the fusion engine understands. The new threads that are spawned can start at either of the following three functions, 'recv_HCT_data', 'recv_HFT_data', or 'recv_ADS_data' depending on the port the parent thread is listening on. This new thread will be passed the master socket, and from it the thread will be able to accept one pending connection. If there are more pending connections the parent thread will span as many threads as necessary in odder to accept all the pending connections. This scheme will enable the fusion engine to handle simultaneously sent data, as each data stream will be processed in a new thread.

Currently, the implementation provides support for HCT, HFT, and ADS data. Each of these three systems sends different data to the fusion engine so each requires a different filter. All messages are sent as ASCII strings (ASCII is used so that we don't have to worry about the Big and Little ending data representations of different systems), with comma delimited fields. In most cases, the data block consists of a header followed by one or more track records. Commas are used to separate individual tracks and the header. At the head of the block is an 8 bit (this is a chosen value) string, which is a number, indicating the length of the block. (The length of the block is required for the recv socket function.) The expected data formats are described below.

\section{HCT DATA:}

The data sent to the fusion engine from an HCT System is not very detailed; currently it contains only the bare minimum that is required to do fusion. The header contains 10 fields and each track record contains 10 fields.

The header contains repeated data as well as control information for the fusion engine. The header is structured as follows:

Table 1: HCT Data Header

\begin{tabular}{|c|c|l|c|}
\hline Data & Type & \multicolumn{1}{|c|}{ Description } & Range \\
\hline Number of tracks & unsigned int & The number of tracks in the data block & n/a \\
\hline Year & unsigned int & 4 digit year & $1-12$ \\
\hline Month & unsigned int & & $1-31$ \\
\hline Day & unsigned int & & $0-23$ \\
\hline Hours & unsigned int & & $0-59$ \\
\hline Minutes & unsigned int & & $0-59$ \\
\hline Seconds & unsigned int & & $1-4096$ \\
\hline Radar Source & unsigned int & The radar ID & S \\
\hline Target Type & Char & S (ship) & 1 \\
\hline
\end{tabular}


The data sent by the HF system is very detailed. It contains many fields that aren't required by the fusion. The header contains 14 fields and each track record contains 24 fields.
The header contains fusion engine control information and repeated data. The header is structured as follows:

Table 2: HFSWR Data Header

\begin{tabular}{|c|c|l|c|}
\hline Data & \multicolumn{1}{|c|}{ Type } & \multicolumn{1}{|c|}{ Description } & Range \\
\hline Scan number & unsigned short & The current scan number & $1-65000$ \\
\hline Number of tracks & unsigned short & Number of tracks in block & $0-35535$ \\
\hline Year & unsigned short & 4 digit year & n/a \\
\hline Month & int & & $1-12$ \\
\hline Day & int & & $1-31$ \\
\hline Hour & int & & $0-23$ \\
\hline Minutes & int & & $0-59$ \\
\hline Seconds & int & & $0-59$ \\
\hline Dwell & float & A unique ID for this sensor & n/a \\
\hline Source ID & int & $1 \rightarrow$ ship target, $2 \rightarrow$ air target & $0-4096$ \\
\hline Target type & int & Measured in meters & 1,2 \\
\hline Range resolution & unsigned short & Measured in degrees & $1500-2000$ \\
\hline Azimuth resolution & int & Measure in meters/second & $5-30$ \\
\hline Doppler resolution & float & Men & $0-10$ \\
\hline
\end{tabular}

\section{ADS DATA:}

The ADS data is special in that it doesn't use a header. A header is not required, as there is no repeated information, and because the fusion engine does not require control information to process the data. The individual ADS tracks are also very brief. The structure of the individual tracks is shown below.

Table 3: ADS Data

\begin{tabular}{|c|l|l|c|}
\hline Data & Type & \multicolumn{1}{|c|}{ Description } & Range \\
\hline Target ID & unsigned int & The ship ID, can be used to tell the ship type & n/a \\
\hline Year & unsigned int & 4 digit year & $1-12$ \\
\hline Month & unsigned int & & $1-31$ \\
\hline Day & unsigned int & & $0-23$ \\
\hline Hour & unsigned int & & $0-59$ \\
\hline Minute & unsigned int & & n/a \\
\hline Latitude & unsigned int & Latitude format: ddmmss - degrees/minutes/seconds & n/a \\
\hline Longitude & unsigned int & Longitude format: ddmmss - degrees/minutes/seconds & $0-359$ \\
\hline Course & unsigned int & Measured in degrees from true North & \\
\hline Speed & unsigned int & Measure in nautical miles & \\
\hline
\end{tabular}

In each ADS track the speed and course are multiplied by 10000 , so before storing the data divide these two values by 10000 to obtain the actual information. The latitude and longitude must also be converted to a degree representation so that they will be compatible with the HCT and HFSWR data.
Once data from a sensor is available, it has to be filtered and stored into a common data structure. The data structure has enough fields to store all the data sent in each HFSWR track.

The below table describes some of the key variables that are used for fusion. 
Table 4: Description of Some Variables

\begin{tabular}{|c|c|}
\hline Variable & Description \\
\hline sensorID & $\begin{array}{l}\text { This is a dynamic array of integers. At input time it should be allocated to size 1, and } \\
\text { initialized with a sensor ID that will be assigned by the fusion engine when } \\
\text { 'getSensorID }(. . .) \text { ' is called. The id range is } 0 \text { to MAX_NUMBER_OF_SESNORS-1. }\end{array}$ \\
\hline targetID & $\begin{array}{l}\text { This is a dynamic array of integers. At input time this array should be allocated with } \\
\text { size } 1 \text {, and initialized with the ID of the track that was sent to the fusion engine. The } \\
\text { ID is not assigned by the fusion engine but by each individual sensor (NOTE: the IDs } \\
\text { from different sensor might not be unique). }\end{array}$ \\
\hline numIDs & This is an integer, which will indicate the size of the sensorID and targetID arrays. \\
\hline fusedID & $\begin{array}{l}\text { This is a unique ID assigned by the fusion engine to each track. The 'fused_do_ids' } \\
\text { function will associate a new ID to each track based on the previous ID associations. }\end{array}$ \\
\hline latitude & $\begin{array}{l}\text { A coordinate representing the location of the target in the North South direction. This } \\
\text { value is measured in degrees ranging from }-90 \text { to } 90 \text {. }\end{array}$ \\
\hline longitude & $\begin{array}{l}\text { A coordinate representing the location of the target in the East West direction. This } \\
\text { value is measured in degrees ranging from }-180 \text { to } 180 \text {. }\end{array}$ \\
\hline speed & The speed of the target in knots. It is only the magnitude. \\
\hline course & Measured from the true North. Values range from 0 to 360 degrees. \\
\hline hours & \multirow{3}{*}{ Time of target detection. } \\
\hline minutes & \\
\hline seconds & \\
\hline
\end{tabular}

\section{Features of the IMS Architecture}

\section{- An Open System}

The design of the proposed architecture concept, i.e., both local IMS and regional IMS incorporates the so-called wide-area surveillance are considered. The advantage is that the

\section{IMS Hierarchy}

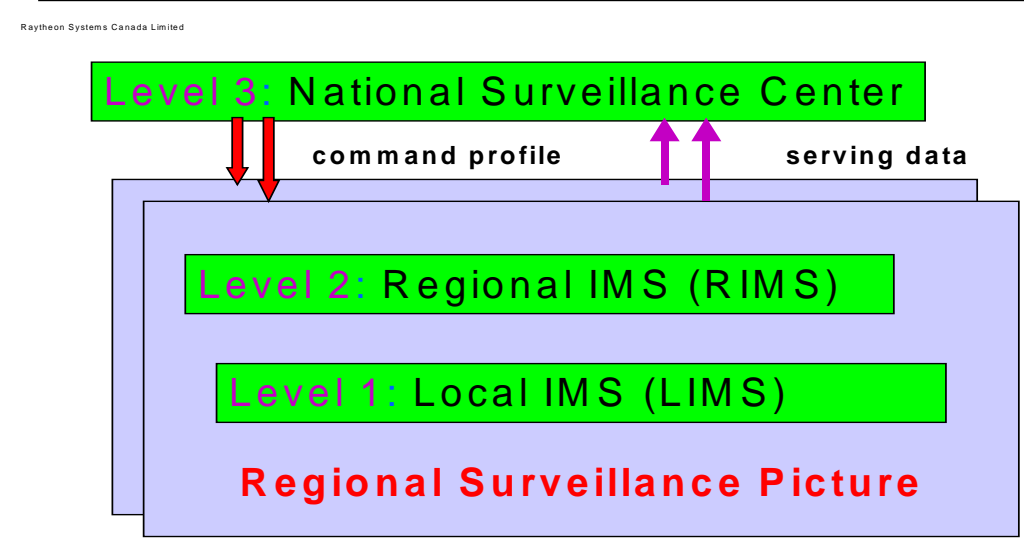

Figure 3: The Proposed IMS Hierarchy 
architecture could be easily configured for a LIMS system or a RIMS system . As a result, we propose the above IMS hierarchy where the LIMS system is structured as follows:

\section{Local IMS Architecture (LIMS)}

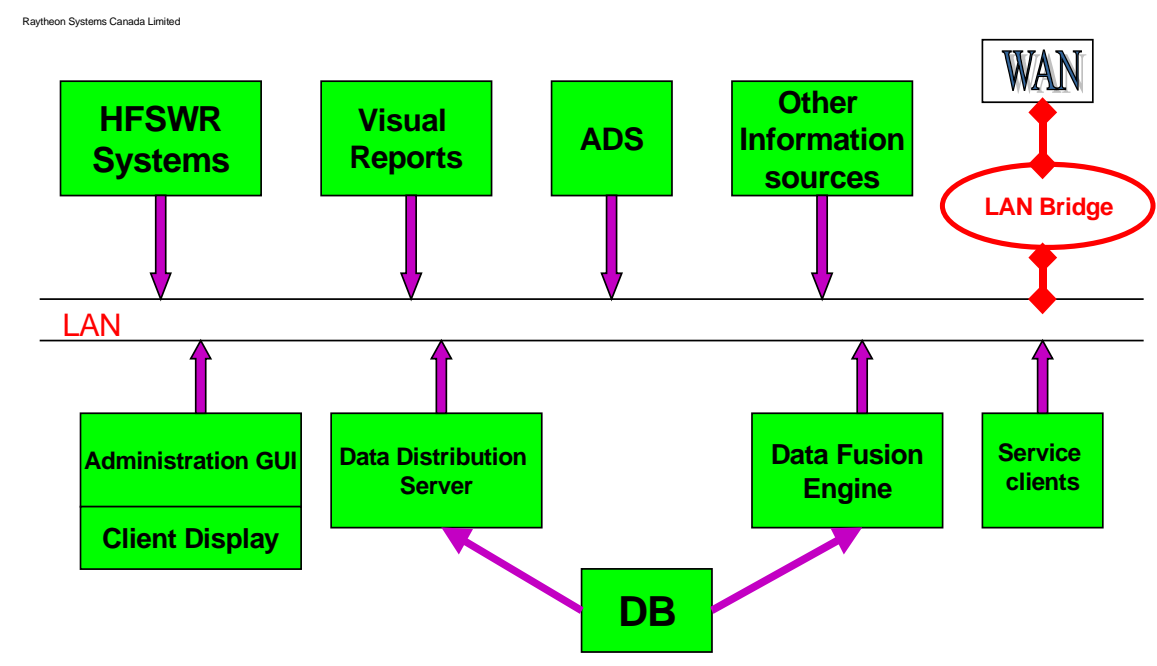

Figure 4: Local IMS

A regional IMS system can be built to coordinate several LIMS systems:

\section{Regional IMS Architecture (RIMS)}

Raytheon Canada Limited

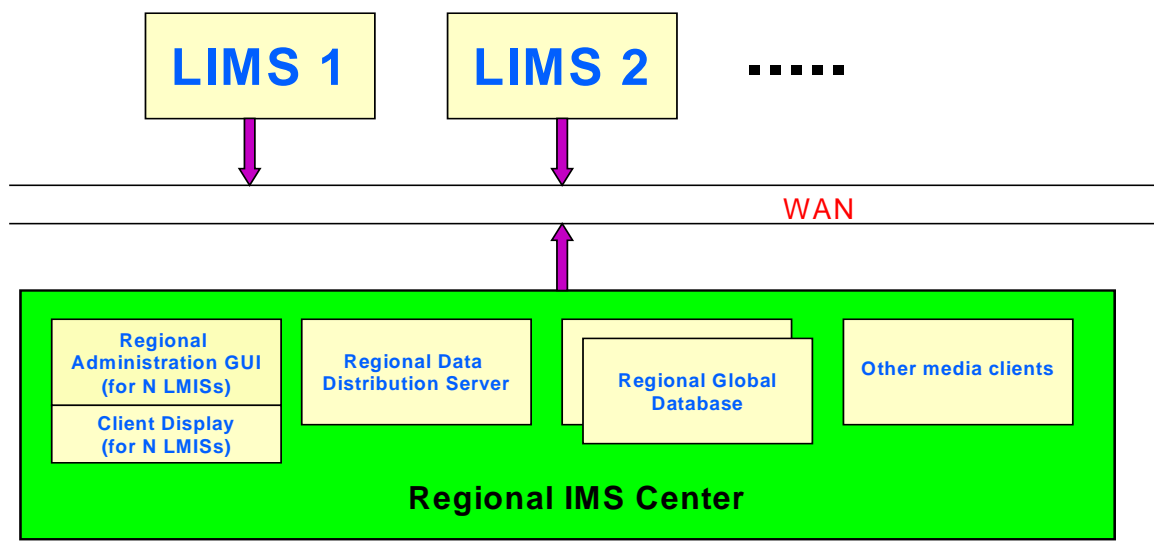

Figure 5: Regional IMS 


\section{- A Network-Centric System}

The network-centric features enable collaborative sensing networks such as:

1. Application layer self-organization

2. Automatic acquisition of context

3. Recruiting and matching sensors to targets

4. Sensor and network resource management

5. Interoperability and transparent access

\section{- A 3-tier System}

The proposed architecture will be a 3-tier system. A general three-tier system will be implemented in the LIMS or RIMS systems. The three-tiers are presentation tier (client), service tier (server) and data tier (database). The separation of the three tiers makes the system easier to maintain, more robust to avoid system crash and securer for the system logic and data.

\section{- A Re-configurable System}

1. The system can be re-configured to use different processing schemes.

2. The system can accept command profiles.

3. The system should be adaptive to environment conditions as well.

\section{- A System with Intelligent Agents Feature}

Intelligent Agents is a flexible infrastructure applicable in many computing environments. Agents are excellent in both interacting with humans and in modeling human behavior, and they offer large advantages of productivity, comprehensibility and reliability in the development of complex, distributed and real time software systems.

In the proposed architecture for IMS system, an intelligent agent can be launched when a special mission is required. For example an agent tracker can be launched for ice-burg tracking in a specific area.

\section{- A System with Recovery, Debugging} and Playback Facilities

Disaster recover, debugging and playback of the system are required. These facilities will be helpful for the development and later-on operation after deployed.

\section{- A system with Diagnosis and Prognosis}

1. The system will provide different log files for status diagnosis and prognosis.

2. The system will provide visualized diagnosis and prognosis information.

\section{Conclusion}

Based on Raytheon Canada's existing HFSWR system, we proposed an architecture for an integrated maritime surveillance (IMS) system to support different needs. Specifically, we discussed the features needed for the proposed architecture. Both LIMS and RIMS are presented for wide-area surveillance. The proposed architecture and its implementation will enhance the capability of the existing system with $\mathrm{HF}$ radars on the Canadian east coast and provide flexible integrated maritime surveillance systems for coasts of different countries.

\section{References}

[1] A. Ponsford, "Surveillance of the 2000 Nautical Mile EEZ Using HFSWR", Canadian Journal of Remote Sensing, Vol. 27, No. 4, 2001.

[2] L. Sevgi, A. Ponsford and H. C. Chan, "An Integrated Maritime Surveillance System Based on High-Frequency Surface-Wave Radars", IEEE Antennas and Propagation Magazine, Vol. 43(4), pp. 28 - 43, August 2001.

[3] L. Sevgi and A. Ponsford, "An HF Radar Based Integrated Maritime Surveillance System", The 3rd International Conference on Circuits, Systems Communication and Computers, Athens, Greece, July 4-8, 1999.

[4] "Data Fusion Processor Enhancement for Coastal Surveillance Network", Technical Report, Raytheon Canada Ltd., ASD-240, ON, Canada, 1995. 
[5] "Canadian East Coast Surveillance System", Technical Report, Raytheon Canada Ltd, RCL7401-1, ON, Canada, 1997.

[6] Z. Ding and K. Hickey, "A Multiple Hypotheses Tracker for a Multiple Sensor Integrated Maritime Surveillance System", Proceeding of the 2nd International Conference on Information Fusion, San Jose, July 6-8, 1999.

[7] E. Durfee, "Strategies for Discovering Coordination Needs in Multi-Agent Systems", Software Tech News, Vol. 5, No. 1, 2002.

[8] I. Jaynes, “Tracking Data Server for Data Fusion and Dissemination", 2001 ONDCP International Technology Symposium, Town and Country Resort, San Diego, CA June 25-28, 2001.

[9] O. Spinczyk, A. Gal, W. SchröderPreikschat, "A Lightweight Component Architecture for Efficient Information Fusion", Proceedings of the International Workshop on Information Fusion (DBFusion 2001), Magdeburg, Deutschland, 9-10 April 2001.

[10] G. Loy, Luke Fletcher, Nicholas Apostoloff, and Alexander Zelinsky, "An Adaptive Fusion Architecture for Target Tracking", International Conference on Automatic Face and Gesture Recognition (FG02), Holiday Inn Capital, $550 \mathrm{C}$ Street N.W., Washington, DC, USA, 20-21 May 2002.

[11] D. Strömberg, “A Sensor-independent Sensor-Oriented Tracking Architecture", Proceedings of Information Decision and Control, Adelaide, Australia, February 1999.

[12] "Software System Architecture", Software System Architecture Lecture Notes, Lucent Technologies, 2001.

[13] É. Bossé, J. Roy and S. Paradis, "Modeling and Simulation in Support of the Design of a Data Fusion System", Journal of Information Fusion, Volume 1, Issue 2, pp. 77-87, December 2000.

[14] A.M. Ponsford, R. Dizaji and Z. Ding, "An HF Surface Wave Radar based Integrated Maritime Surveillance System", The 34-th International Symposium METRA, ROMANIA, May 29-30, 2003. 